\title{
Considerations concerning the generalization of the Dirac equations to unstable fermions
}

\author{
Bernd A. Kniehl ${ }^{*}$ and Alberto Sirlin ${ }^{\dagger}$ \\ Max-Planck-Institut für Physik (Werner-Heisenberg-Institut), Föhringer Ring 6, 80805 Munich, Germany \\ (Received 12 August 2014; published 6 October 2014)
}

\begin{abstract}
We discuss the generalization of the Dirac equations and spinors in momentum space to free unstable spin-1/2 fermions taking into account the fundamental requirement of Lorentz covariance. We derive the generalized adjoint Dirac equations and spinors, and explain the very simple relation that exists, in our formulation, between the unstable and stable cases. As an application of the generalized spinors, we evaluate the probability density. We also discuss the behavior of the generalized Dirac equations under time reversal.
\end{abstract}

DOI: 10.1103/PhysRevD.90.077901

The necessity to generalize the Dirac equations and spinors in momentum space to free unstable spin- $1 / 2$ particles has recently been recognized in connection with the wave-function renormalizations of mixed systems of Dirac [1,2] and Majorana fermions [3]. In this paper, we discuss their construction when the fundamental requirement of Lorentz covariance is taken into account. We also derive the generalized adjoint Dirac equations and spinors, and explain the very simple relation that exists, in our formulation, between the generalized Dirac equations and spinors and the corresponding expressions for stable fermions. ${ }^{1}$ We illustrate the application of the generalized spinors by evaluating the probability density.

Defining the complex mass $M$ of the unstable fermion as the zero of the inverse propagator, a frequently used parametrization is [4]

$$
M=m-i \frac{\Gamma}{2},
$$

where $m$ and $\Gamma$ are its mass and width, respectively.

We define the four-momentum of the unstable fermion according to

$$
p^{0}=M \gamma c, \quad \vec{p}=M \gamma \vec{v},
$$

where $\gamma=\left(1-\vec{v}^{2} / c^{2}\right)^{-1 / 2}$ and $\vec{v}$ is the particle's velocity in the chosen inertial frame. Since Eq. (2) differs from the expressions of special relativity for stable fermions by only the constant factor $M / m, p^{\mu}=\left(p^{0}, \vec{p}\right)$ transforms as a four-vector. Equation (2) can also be written as

\footnotetext{
*Permanent address: II. Institut für Theoretische Physik, Universität Hamburg, Luruper Chaussee 149, 22761 Hamburg, Germany. kniehl@desy.de

${ }^{\dagger}$ Permanent address: Department of Physics, New York University, 4 Washington Place, New York, NY 10003, USA. alberto.sirlin@nyu.edu

${ }^{1}$ For brevity, spin- $1 / 2$ particles are henceforth called fermions.
}

PACS numbers: 03.65.Pm, 11.30.Er, 12.15.Ff, 12.15.Lk

$$
p^{\mu}=M u^{\mu},
$$

where $u^{\mu}=\gamma(c, \vec{v})$ is the four-velocity. From Eqs. (2) and (3) one finds the basic relation ${ }^{2}$

$$
p_{\mu} p^{\mu}=p^{02}-\vec{p}^{2}=M^{2} c^{2} .
$$

Evaluating the real and imaginary parts of $p^{0}$ from Eq. (4) and using the relation $\operatorname{Im} \vec{p}^{2}=-\left[m \Gamma /\left(m^{2}-\Gamma^{2} / 4\right)\right] \operatorname{Re} \vec{p}^{2}$ that follows from Eqs. (1) and (2), one can express $p^{0}$ in terms of $\operatorname{Re} \vec{p}^{2}, m$, and $\Gamma$ as

$$
p^{0}=M\left[\frac{\operatorname{Re} \vec{p}^{2}+\left(m^{2}-\Gamma^{2} / 4\right) c^{2}}{m^{2}-\Gamma^{2} / 4}\right]^{1 / 2} .
$$

In the limit $\Gamma \rightarrow 0, \vec{p}^{2}$ is real, $p^{0}=E / c$, and Eq. (5) becomes

$$
E=\left(\vec{p}^{2} c^{2}+m^{2} c^{4}\right)^{1 / 2},
$$

the well-known energy-momentum relation for stable particles. In the rest frame, $\vec{p}=\overrightarrow{0}$ and Eq. (5) reduces to $p^{0}=M c$, in agreement with Eq. (2) when $\vec{v}=\overrightarrow{0}$.

In momentum space, the generalizations of the Dirac equations to free unstable fermions are

$$
(\not p-M c) u_{r}(\vec{p})=0, \quad(\not p+M c) v_{r}(\vec{p})=0,
$$

where $\not p=p_{\mu} \gamma^{\mu}$ and $r=1,2$ labels the two independent solutions. Recalling Eq. (2), we note that Eq. (7) can be derived by multiplying the corresponding Dirac equations for stable fermions by $M / m$. The four independent solutions can be written explicitly in the form

\footnotetext{
${ }^{2}$ In this paper, we adopt the notations and conventions of Refs. [5,6].
} 


$$
\begin{aligned}
& u_{r}(\vec{p})=\left(\frac{p^{0}+M c}{2 M c}\right)^{1 / 2}\left(\begin{array}{c}
\frac{\chi_{r}}{\frac{\vec{\sigma} \cdot \vec{p}}{p^{0}+M c}} \chi_{r}
\end{array}\right), \\
& v_{r}(\vec{p})=\left(\frac{p^{0}+M c}{2 M c}\right)^{1 / 2}\left(\begin{array}{c}
\frac{\vec{\sigma} \cdot \vec{p}}{p^{0}+M c} \chi_{r}^{\prime} \\
\chi_{r}^{\prime}
\end{array}\right),
\end{aligned}
$$

where $\sigma^{i}$ are the Pauli matrices and $\chi_{r}$ and $\chi_{r}^{\prime}$ are twodimensional constant and orthogonal spinors frequently chosen as

$$
\chi_{1}=\chi_{2}^{\prime}=\left(\begin{array}{l}
1 \\
0
\end{array}\right), \quad \chi_{2}=\chi_{1}^{\prime}=\left(\begin{array}{l}
0 \\
1
\end{array}\right) .
$$

With this choice, $u_{r}(\vec{p})$ and $v_{r}(\vec{p})$ are eigenstates of the $z$ component of spin in the rest frame of the fermion, with eigenvalue $+\hbar / 2$ (spin up) for $u_{1}(\vec{p})$ and $v_{2}(\vec{p})$ and $-\hbar / 2$ (spin down) for $u_{2}(\vec{p})$ and $v_{1}(\vec{p})$.

Including the space-time dependencies, the plane-wave solutions associated with the spinors $u_{r}(\vec{p})$ and $v_{r}(\vec{p})$ are $u_{r}(\vec{p}) \exp (-i p \cdot x)$ and $v_{r}(\vec{p}) \exp (i p \cdot x)$, respectively. Using Eqs. (1)-(3), we have

$$
e^{-i p \cdot x}=e^{-i m u \cdot x} e^{-(\Gamma / 2) u \cdot x} .
$$

The first factor on the right-hand side of Eq. (10) is the space-time dependence in the stable case, while the second factor reflects the fact that the fermion is unstable. The amplitude $u_{r}(\vec{p}) \exp (-i m u \cdot x)$ is a solution of the usual Dirac equation for stable fermions and is, therefore, time-reversal invariant. By contrast, the second factor, $\exp \left[-\Gamma \gamma\left(c^{2} t-\vec{v} \cdot \vec{x}\right) / 2\right]$, is not invariant under the time-reversal transformation $t \rightarrow-t$ and $\vec{v} \rightarrow-\vec{v}$.

Another simple way to show that the generalized Dirac equations are not invariant under time reversal is the following: we recall that the operator that relates the wave functions at times $t$ and $t^{\prime}=-t$ is antiunitary, namely of the form $K U$, where $U$ is a unitary matrix and $K$ means complex conjugation. If the Hamiltonian $H(t)$ at time $t$ involves the complex mass $M$, as is the case in the formulation of the generalized Dirac equations, when $K$ acts on $H(t)$ it transforms $M \rightarrow M^{*}$. As a consequence, $H\left(t^{\prime}\right)$ differs from $H(t)$ by the same change $M \rightarrow M^{*}$, and the proof of time-reversal invariance, explained for instance in Ref. [5], breaks down.

The Hermitian adjoints of Eq. (7) are

$$
\bar{u}_{r}(\vec{p})\left(\not p^{*}-M^{*} c\right)=0, \quad \bar{v}_{r}(\vec{p})\left(\not p^{*}+M^{*} c\right)=0,
$$

where

$$
\bar{u}_{r}(\vec{p})=u_{r}^{\dagger}(\vec{p}) \gamma^{0}, \quad \bar{v}_{r}(\vec{p})=v_{r}^{\dagger}(\vec{p}) \gamma^{0}
$$

are the usual adjoint spinors and $p^{*}=p_{\mu}^{*} \gamma^{\mu}$. At first sight, the presence of the complex conjugates $p_{\mu}^{*}$ and $M^{*}$ seems to complicate matters. However, we note from Eq. (3) that $p^{\mu} / M=u^{\mu}$ is real. Therefore, we have the important relation

$$
\left(\frac{p^{\mu}}{M}\right)^{*}=\frac{p^{\mu}}{M}
$$

Inserting $p_{\mu}^{*}=\left(M^{*} / M\right) p_{\mu}$ and multiplying by $M / M^{*}$, Eq. (11) becomes

$$
\bar{u}_{r}(\vec{p})(\not p-M c)=0, \quad \bar{v}_{r}(\vec{p})(\not p+M c)=0 .
$$

The four generalized Dirac equations shown in Eqs. (7) and (14) were postulated in Ref. [2] without applying Eq. (13) and with a different interpretation of the adjoint spinors $\bar{u}_{r}(\vec{p})$ and $\bar{v}_{r}(\vec{p})$. In the case of unstable fermions, Eqs. (7) and (14) play an important role in the implementation of the Aoki-Hioki-Kawabe-Konuma-Muta (AHKKM) [7] renormalization conditions in general theories with intergeneration mixing, as pointed out in Refs. [1-3].

Since $p^{\mu}$ transforms as a four-vector, the proof of Lorentz covariance of Eq. (7) follows the same steps as the proof of the Lorentz covariance of the Dirac equation in coordinate space (see, for example, chapter 2 in Ref. [5]). Specifically, if $p^{\mu}$ and $u_{r}(\vec{p})$ are the four-momentum and the spinor in the Lorentz frame $O$ and $p^{\prime \mu}$ and $u_{r}^{\prime}\left(\vec{p}^{\prime}\right)$ are those in the Lorenz frame $O^{\prime}$, one expresses, for example, the first equality in Eq. (7) in terms of the $O^{\prime}$ variables by means of the relations $p_{\mu}=a^{\nu}{ }_{\mu} p_{\nu}^{\prime}$, where $a^{\nu}{ }_{\mu}$ are the coefficients of the Lorentz transformation between the fourvectors $p_{\mu}$ and $p_{\mu}^{\prime}$, and $u_{r}(\vec{p})=S^{-1} u_{r}^{\prime}\left(\vec{p}^{\prime}\right)$, where $S$ is a matrix that satisfies the relations $a^{\nu}{ }_{\mu} S \gamma^{\mu} S^{-1}=\gamma^{\nu}$ and $S^{-1}=\gamma^{0} S^{\dagger} \gamma^{0}$. Then the first equality in Eq. (7) becomes $\left(\not \not^{\prime}-M c\right) u_{r}^{\prime}\left(\vec{p}^{\prime}\right)=0$, which demonstrates its Lorentz covariance. Carrying out the Hermitian conjugation of the $O^{\prime}$ Dirac equation and using Eq. (13), one finds $\bar{u}_{r}^{\prime}\left(\vec{p}^{\prime}\right)\left(\not p^{\prime}-M c\right)=0$, which shows the Lorentz covariance of the corresponding adjoint Dirac equation, Eq. (14).

The adjoint spinors with respect to $u_{r}(\vec{p})$ and $v_{r}(\vec{p})$ in Eq. (8) are

$$
\begin{aligned}
& \bar{u}_{r}(\vec{p})=\left(\frac{p^{0}+M c}{2 M c}\right)^{1 / 2}\left(\chi_{r}^{\dagger},-\chi_{r}^{\dagger} \frac{\vec{\sigma} \cdot \vec{p}}{p^{0}+M c}\right), \\
& \bar{v}_{r}(\vec{p})=\left(\frac{p^{0}+M c}{2 M c}\right)^{1 / 2}\left(\chi_{r}^{\prime \dagger} \frac{\vec{\sigma} \cdot \vec{p}}{p^{0}+M c},-\chi_{r}^{\prime \dagger}\right),
\end{aligned}
$$

where we have again applied Eq. (13) to eliminate $p_{\mu}^{*}$ and $M^{*}$. In particular, Eq. (13) implies that $\left[\left(p^{0}+M c\right) /\right.$ $(2 M c)]^{1 / 2}$ and $\vec{p} /\left(p^{0}+M c\right)$ are real.

It is interesting to note that the four generalized Dirac equations shown in Eqs. (7) and (14) as well as their spinor solutions presented in Eqs. (8) and (15) can be obtained from the corresponding ones for stable fermions, for $\Gamma=0$, by simply substituting $m \rightarrow M$ in their explicit mass dependencies and in the definition of $p^{\mu}$ in Eqs. (2) and (3).

Since $M$ cancels in the ratios $\left(p^{0}+M c\right) /(2 M c)$ and $\vec{p} /\left(p^{0}+M c\right)$, these factors are the same as in the $\Gamma=0$ case. It hence follows that the spinor solutions in Eqs. (8) and (15) satisfy the same normalization and completeness 
relations as in the case of stable fermions, which are given, for example, by Eqs. (A.29) and (A.30) in Ref. [6]. In particular,

$$
\begin{aligned}
& \bar{u}_{r}(\vec{p}) u_{s}(\vec{p})=-\bar{v}_{r}(\vec{p}) v_{s}(\vec{p})=\delta_{r s}, \\
& \bar{u}_{r}(\vec{p}) v_{s}(\vec{p})=-\bar{v}_{r}(\vec{p}) u_{s}(\vec{p})=0 .
\end{aligned}
$$

In some applications, the two-component spinors $\chi_{r}$ and $\chi_{r}^{\prime}$ in Eq. (9) are replaced by helicity eigenstates $\phi_{s}$ and $\phi_{s}^{\prime}$, respectively. Because, in the case of unstable particles, $\vec{p}$ is complex [cf. Eqs. (2) and (3)], the usual helicity projection,

$$
\frac{\vec{p}}{|\vec{p}|} \cdot \frac{\vec{\sigma}}{2} \phi_{s}=s \phi_{s}, \quad \frac{\vec{p}}{|\vec{p}|} \cdot \frac{\vec{\sigma}}{2} \phi_{s}^{\prime}=-s \phi_{s}^{\prime},
$$

where $|\vec{p}|=\left(\vec{p}^{*} \cdot \vec{p}\right)^{1 / 2}$, leads to complex eigenvalues $s= \pm\left(\vec{p}^{2}\right)^{1 / 2} /(2|\vec{p}|)$, as can be checked by applying the helicity projection operator twice. A consistent alternative is to define the helicity projection operator in Eq. (17) according to $\vec{u} /|\vec{u}| \cdot \vec{\sigma} / 2$, where $\vec{u}=\vec{p} / M$ are the spatial components of the four-velocity $u$ given below Eq. (3), or, equivalently, $\vec{v} /|\vec{v}| \cdot \vec{\sigma} / 2$.

It is also instructive to calculate the probability current using the spinor solutions in Eqs. (8) and (15). We find

$$
c \bar{u}_{r}(\vec{p}) \gamma^{\mu} u_{r}(\vec{p})=c \bar{v}_{r}(\vec{p}) \gamma^{\mu} v_{r}(\vec{p})=\frac{p^{\mu}}{M}=u^{\mu}
$$

As expected, these currents transform as four-vectors. In particular, for $\mu=0$ we have

$$
c u_{r}^{\dagger}(\vec{p}) u_{r}(\vec{p})=c v_{r}^{\dagger}(\vec{p}) v_{r}(\vec{p})=u^{0}=\gamma c .
$$

Since $c u_{r}^{\dagger}(\vec{p}) u_{r}(\vec{p})$ and $c v_{r}^{\dagger}(\vec{p}) v_{r}(\vec{p})$ are the probability densities, they should be real and positive, consistent with Eq. (19).

Another interesting application is to examine the spacetime dependence of the probability density. Incorporating the space-time factor $\exp (-i p \cdot x)$ of Eq. (10) in $c u_{r}^{\dagger}(\vec{p}) u_{r}(\vec{p})$, we find that the latter is multiplied by an overall factor $\exp \left[-\Gamma \gamma\left(c^{2} t-\vec{v} \cdot \vec{x}\right)\right]$, which implies that the probability density for the positive-energy states decreases exponentially with time, reflecting the fermion's instability.

In summary, (i) we have proposed a simple definition of the (complex) four-momentum of a free unstable spin-1/2 particle [cf. Eqs. (2) and (3)] and shown that it indeed transforms as a four-vector, (ii) we have derived the generalized Dirac equations in momentum space [cf. Eq. (7)] and found explicit spinor solutions [cf. Eq. (8)], (iii) we have derived the generalized adjoint Dirac equations [cf. Eq. (14)] and spinors [cf. Eq. (15)] by Hermitian conjugation of Eqs. (7) and (8), respectively, taking into account the basic relation in Eq. (13), (iv) using the important fact that $p^{\mu}$ transforms as a four-vector, we have shown how the proof of Lorentz covariance carries over to the generalized Dirac equations and their adjoints, (v) we have pointed out the very simple relation that exists, in our formulation, between the generalized Dirac equations and spinors and the corresponding expressions for stable fermions, (vi) in particular, we have shown that our spinors and adjoint spinors satisfy the same normalization and completeness relations as in the case of stable fermions, (vii) we have proposed a modified definition of the helicity projection operator for unstable fermions that leads to real eigenvalues, (viii) as an illustration, we have applied our spinors and adjoint spinors to calculate the probability density and found that it satisfies the expected theoretical properties, and (ix) we have discussed the behavior of the generalized Dirac equations under time reversal. As mentioned after Eq. (14), the four generalized Dirac equations in Eqs. (7) and (14) play an important role in the implementation of the AHKKM [7] renormalization conditions for unstable fermions in general theories with intergeneration mixing.

We thank the Werner-Heisenberg-Institut for the hospitality extended to us during a visit when this paper was prepared. This research was supported in part by the German Research Foundation through the Collaborative Research Center No. 676 Particles, Strings and the Early Universe-The Structure of Matter and Space Time. The work of A. S. was supported in part by the National Science Foundation through Grant No. PHY-1316452.
[1] B. A. Kniehl and A. Sirlin, Phys. Rev. D 77, 116012 (2008); 85, 036007 (2012); B. A. Kniehl, Phys. Rev. Lett. 112, 071603 (2014).

[2] B. A. Kniehl, Phys. Rev. D 89, 096005 (2014).

[3] B. A. Kniehl, Phys. Rev. D 89, 116010 (2014).

[4] M. C. Smith and S. S. Willenbrock, Phys. Rev. Lett. 79, 3825 (1997); M. Passera and A. Sirlin, Phys. Rev. D 58, 113010 (1998).
[5] J.D. Bjorken and S.D. Drell, Relativistic Quantum Mechanics (McGraw-Hill, New York, 1964).

[6] F. Mandl and G. Shaw, Quantum Field Theory (Wiley, Chichester, 1984).

[7] K.-i. Aoki, Z. Hioki, R. Kawabe, M. Konuma, and T. Muta, Prog. Theor. Phys. Suppl. 73, 1 (1982). 